\title{
Degradation of acetalated dextran can be broadly tuned based on cyclic acetal coverage and molecular weight
}

\author{
Naihan Chen ${ }^{\mathrm{a}}$, Michael A. Collier ${ }^{\mathrm{a}}$, Matthew D. Gallovic ${ }^{\mathrm{a}, \mathrm{b}}$, Graham C. Collins $^{\mathrm{a}}$, \\ Carla C. Sanchez ${ }^{\mathrm{a}}$, Elizabeth Q. Fernandes ${ }^{\mathrm{a}}$, Eric M. Bachelder ${ }^{\mathrm{a}}$, \\ Kristy M. Ainslie, Associate Professor ${ }^{\mathrm{a}, *}$ \\ a Division of Molecular Pharmaceutics, Eshelman School of Pharmacy, The University of North Carolina at Chapel Hill, NC, USA \\ ${ }^{\mathrm{b}}$ Department of Chemical and Biomolecular Engineering, College of Engineering, The Ohio State University, Columbus, OH, USA
}

\section{A R T I C L E I N F O}

\section{Article history:}

Received 23 May 2016

Received in revised form 9 August 2016

Accepted 15 August 2016

Available online 16 August 2016

\section{Keywords:}

Acid sensitive polymer

Tunable degradation

Temporal drug release

Drug delivery

Resiquimod

Particle formulation

\begin{abstract}
A B S T R A C T
Microparticles (MPs) derived from acid-sensitive biopolymers enable rapid degradation and cargo release under acidic conditions, such as at tumor microenvironments, within lysosomal/phagosomal compartments inside phagocytic cells, or at sites of inflammation. One such acid-sensitive biopolymer, acetalated dextran (Ace-DEX), has tunable degradation rates and $\mathrm{pH}$-neutral degradation byproducts consisting of dextran, acetone, and ethanol. By studying the degradation profiles of Ace-DEX MPs with varying cyclic acetal coverage (CAC) and dextran molecular weight (MW), we concluded that MPs composed of low CAC or high MW polymer degraded the fastest at both $\mathrm{pH} 7.4$ and 5.0. To further understand the properties of this unique polymer, we encapsulated a model drug resiquimod, which is a toll-like receptor (TLR) 7/8 agonist, into Ace-DEX MPs of different polymer CAC and dextran MW. It was observed that resiquimod was released faster from MPs of lower CAC or higher MW. By evaluating the activation of RAW macrophages cultured with different types of resiquimod-loaded Ace-DEX MPs, we found that MPs of lower CAC or higher MW promoted greater nitrite production and resulted in more robust cell activation. Our results indicate we can precisely control the degradation profile, release kinetics, and bioactivity of encapsulated cargos by altering CAC and MW, furthering Ace-DEX MPs' novelty as a drug carrier.
\end{abstract}

(c) 2016 Elsevier B.V. All rights reserved.

\section{Introduction}

Acid-sensitive polymers are widely studied and of increasing interest for drug delivery applications (Jeong and Gutowska, 2002).

Abbreviations: MPs, microparticles; MHC, major histocompatibility complex; PBAEs, poly( $($ amino) esters; POEs, poly(ortho esters); APCs, antigen presenting cells; PLGA, poly(lactic-co-glycolic acid); Ac-DEX, methoxy acetal derivatized acetalated dextran; Ace-DEX, ethoxy acetal derivatized acetalated dextran; CAC, cyclic acetal coverage; MW, molecular weight; TEA, triethylamine; NMR, nuclear magnetic resonance; PVA, poly(vinyl alcohol); PBS, phosphate buffered saline; EA, ethyl acetate; DMSO, dimethyl sulfoxide; DLS, dynamic light scattering; SEM, scanning electron microscopy; BCA, bicinchoninic acid based assay; MTT, 3-(4,5dimethylthiazol-2-yl)-2,5-diphenyltetrazolium bromide; LDH, lactate dehydrogenase; LPS, lipopolysaccharide; TLR, toll-like receptor.

* Corresponding author at: UNC Eshelman School of Pharmacy, Division Molecular Pharmaceutics, 4211 Marsico Hall, 125 Mason Farm Road, Chapel Hill, NC 27599, USA.

E-mail address: ainsliek@email.unc.edu (K.M. Ainslie).
Because these polymers are stable at neutral environments and degrade rapidly at lower $\mathrm{pH}$ levels, they can provide triggered release in biological regions of interest where there is an acidic local environment. Several areas of the body can contain lower $\mathrm{pH}$ regions, such as at tumor tissues (Engin et al., 1995; Lee et al., 2008), within intracellular compartments (Hu et al., 2007; Mellman et al., 1986), and at sites of inflammation (Ulbrich and Lamprecht, 2010). Utilizing the natural pH gradient within biological systems, microparticles (MPs) composed of acid-labile polymers can enhance biological responses as it allows for targeted delivery of chemotherapeutic agents to the acidic tumor microenvironment (Lee et al., 2008), increased transfection efficiency of DNA vaccine antigens via the $\mathrm{pH}$-gradient in the endocytic pathway (Little et al., 2004), and localized delivery of immunosuppressants to inflammatory sites of the gastrointestinal tract (Makhlof et al., 2009). Moreover, acid-sensitive polymeric carriers can also reduce the frequency of adjuvant administration (i.e., dose-sparing) (Duong et al., 2013; Peine et al., 2013) and promote 
antigen cross presentation on both major histocompatibility complex (MHC) I and MHC II complexes following phagosomal escape of encapsulated antigens (Broaders et al., 2009), which are both advantageous for vaccine applications.

There are currently a few acid-sensitive polymers under investigation for drug delivery, such as poly(ß-amino) esters (PBAEs), poly(ortho esters) (POEs), and polyketals (Lee et al., 2009; Yang et al., 2008). PBAEs have been developed primarily for DNA vaccine applications (Little et al., 2004, 2005). Due to their acidsensitivity, PBAEs particles dissolve rapidly after being phagocytosed by antigen presenting cells (APCs), leading to an increased transfection efficiency and higher levels of dendritic cell activation compared to poly(lactic-co-glycolic acid) (PLGA) particles. Despite this promising outcome, PBAEs are associated with potential toxicity and may cause severe side effects for long-term treatment (Little et al., 2004, 2005). POEs, on the other hand, have good biocompatibility and degrade rapidly under acidic conditions. However, the ortho ester group hydrolyzes quickly in neutral environments and is stable only under basic conditions ( $\mathrm{pH} 8.4$ ) (Ji et al., 2014a, 2014b). Polyketals display reduced cytotoxicity and have high acid sensitivity as suggested by studies performed using a library of polyurethanes and polyureas containing the same dimethyl ketal moiety (Paramonov et al., 2008). However, all these polymers have complex synthesis reactions, which makes them challenging to be broadly accessible. Therefore, due to the limitations of existing acid-labile polymeric systems, further work must be performed to design an easily synthesized polymer with acid-sensitivity, and minimal toxicity that allows for vaccine and drug delivery.

One alternative acid-sensitive polymer is acetalated dextran. It is synthesized from the dextran polysaccharide with the hydroxyl groups on the glucose backbone replaced by hydrophobic acetals. Because acetal groups are acid sensitive, acetalated dextran hydrolyzes more rapidly under acidic conditions into dextran, acetone, and an alcohol. There are two types of acetalated dextran polymers depending on their degradation products: Ac-DEX (methoxy acetal derivatized acetalated dextran) that produces methanol as the degradation metabolite (Bachelder et al., 2008) and Ace-DEX (ethoxy acetal derivatized acetalated dextran) that produces ethanol (Kauffman et al., 2012a). Unlike PLGA and some polyanhydrides that degrade into acidic byproducts, all degradation metabolites of acetalated dextran (dextran, acetone, and an alcohol) are pH-neutral. Thus, hydrolytic degradation of Ac-DEX or Ace-DEX should not disrupt the local pH environment or harm the encapsulated payload. This is important because acidification of the local microenvironment, as seen with PLGA hydrolysis, may result in tissue toxicity and damage to sensitive cargos (Fu et al., 2000; Na et al., 2003; Sung et al., 2004).

Besides neutral degradation products, acetalated dextran is advantageous also due to its tunable degradation profiles. Depending on the length of the synthesis reaction, varying degrees of cyclic or acyclic acetal groups will form. Cyclic acetal groups, which are the thermodynamic product, form with longer reaction times and exhibit more hydrolytic stability. On the contrary, acyclic acetal groups, which are the kinetic product, form with shorter reaction times and hydrolyze faster. Therefore, the degradation rate of acetalated dextran can be tuned by varying the length of the synthesis reaction. Half-lives of Ac-DEX range from minutes to months (Broaders et al., 2009), with its hydrolysis rate relying on the reaction time and dextran MW (Kauffman et al., 2012b). Precise control of the polymer's degradation profile allows for both burst and sustained cargo release, which makes it an ideal carrier for desired therapies. Its stability outside cold chain conditions serves as another advantage of acetalated dextran. Kanthamneni et al. showed that Ac-DEX MPs containing horseradish peroxidase remained stable when stored at $-20^{\circ} \mathrm{C}, 4^{\circ} \mathrm{C}, 25^{\circ} \mathrm{C}$, or $45^{\circ} \mathrm{C}$ for 3 months (Kanthamneni et al., 2012). This property makes acetalated dextran easy to handle and desirable for use in developing nations.

Micelles and liposomes can be comprised of acid-sensitive polymers, but they may be hard to manufacture in large scale for drug delivery applications (Mandracchia et al., 2004; Murthy et al., 2002; Sawant et al., 2006) and often require cold-chain storage. In contrast, polymeric MPs can be made in a scalable fashion (Duong et al., 2013), have been shown to protect cargos outside the cold chain (Kanthamneni et al., 2012), and can be sized more aptly for passive targeting of relevant immune cells (Manolova et al., 2008). In order to demonstrate an acid-responsive platform that has tunable degradation profiles, we characterized the degradation kinetics of Ace-DEX MPs composed of polymers of different CAC and MWs. First, we analyzed the hydrophobicity of Ace-DEX polymers of varying CAC and MW. The polymers were then formulated into MPs, which were evaluated for size and morphology. The degradation properties of these MPs were investigated for their acid-sensitive degradation characteristics and the ability to tune degradation rate with CAC and MWs. Lastly, we examined the effect of tunable degradation profiles on the release kinetics and bioactivity of encapsulated cargos. Using resiquimod (an intraphagosomal toll-like receptor $7 / 8$ agonist) as a model drug, we characterized the release kinetics of different MP sets and studied the dependence of degradation profiles on drug bioactivity using RAW 264.7 macrophages. Cyto-compatibility of blank and resiquimod-loaded MPs was also assessed. The aim of this study is to characterize the tunable degradation kinetics of the acid-sensitive Ace-DEX polymer based on CAC and MW, and demonstrate how variability in degradation impacts drug bioactivity and cyto-compatability by changing the release kinetics of encapsulated cargos.

\section{Materials and methods}

\subsection{Materials}

All chemicals were purchased from Sigma-Aldrich (St. Louis, MO) and used unmodified unless otherwise noted. Water used in these experiments was purified using a Millipore Milli-Q Integral Water Purification System (Billerica, MA). In the presence of acetalcontaining materials, basic water ( $\mathrm{pH}$ 9.0) was used by the addition of triethylamine (TEA) $(0.01 \% \mathrm{v} / \mathrm{v})$. Resiquimod was purchased from Alexis Biochemicals, Enzo Life Sciences (Farmingdale, NY). Fluorescence and absorbance readings were generated using a Molecular Devices SpectraMax M2 (Sunnyvale, CA).

\subsection{Cell culture}

RAW 264.7 macrophages (ATCC, Manassas, VA) were grown and cultured according to the manufacturer's protocol. Cells were maintained at $37{ }^{\circ} \mathrm{C}$ with $5 \% \mathrm{CO}_{2}$ and $100 \%$ relative humidity. Culture media consisted of Dulbecco's Modified Eagle's Medium (Fischer Scientific, Pittsburgh, PA), 10\% fetal bovine serum (Hyclone, Pittsburgh, PA), and 1\% penicillin-streptomycin (Fischer Scientific, Pittsburgh, PA).

\subsection{Synthesis and CAC analysis of Ace-DEX polymer}

Ace-DEX polymer was synthesized from $10,71,500$, or $2000 \mathrm{kDa}$ dextran with some modifications to the previously described procedure (Kauffman et al., 2012a). To synthesize 10 or $71 \mathrm{kDa}$ Ace-DEX, lyophilized dextran (10 or $71 \mathrm{kDa}$ ) and pyridinium $p$-toluenesulfonate $(0.0617 \mathrm{mmol})$ were dissolved in anhydrous dimethyl sulfoxide (DMSO, $10 \% \mathrm{vol} /$ wt poly). The mixture was reacted with 2-ethoxypropene $(37 \mathrm{mmol}$; Waterstone, Carmel, IN) under nitrogen gas at room temperature. In order to 
obtain $20 \%, 40 \%$, or $60 \%$ CAC, the $10 \mathrm{kDa}$ reaction went for $5.2,52.6$, or $1440 \mathrm{~min}$, while the $71 \mathrm{kDa}$ reaction went for $3.2,25$, or $1560 \mathrm{~min}$. To synthesize 500 or $2000 \mathrm{kDa}$ Ace-DEX, lyophilized dextran (500 or $2000 \mathrm{kDa}$ ) and pyridinium $p$-toluenesulfonate $(0.0308 \mathrm{mmol})$ were dissolved in DMSO $(6.25 \% \mathrm{vol} / \mathrm{wt}$ poly). The mixture was reacted with 2-ethoxypropene $(18.5 \mathrm{mmol})$. To obtain a $20 \%, 40 \%$, or $60 \%$ CAC, the $500 \mathrm{kDa}$ reaction continued for $4.4,10$, or $166 \mathrm{~min}$. The $2000 \mathrm{kDa}$ reaction went for 13.8 or $55.2 \mathrm{~min}$ to achieve a $40 \%$ or $60 \%$ CAC. $2000 \mathrm{kDa} 20 \%$ CAC polymer was reacted, but it could not form particles. At desired time points, the reactions were quenched with TEA. The quenched reactions were precipitated with basic water, centrifuged at $14,400 \mathrm{rpm}$ for $10 \mathrm{~min}$ at $4{ }^{\circ} \mathrm{C}$ (Thermo Scientific Sorvall Legend XTR, Waltham, MA) to remove the supernatant. The resulting pellet was then frozen and lyophilized. To further purify the polymer, the product was dissolved in ethanol (200 proof) and centrifuged at $14,400 \mathrm{rpm}$ for $10 \mathrm{~min}$ at $4{ }^{\circ} \mathrm{C}$. The supernatant was precipitated in basic water and lyophilized for another 2 days to yield Ace-DEX polymer. CAC was determined using $1 \mathrm{H}$ nuclear magnetic resonance (NMR) based on a previously developed method (Bachelder et al., 2008; Kauffman et al., 2012a).

\subsection{Wettability testing using water contact angle assay}

Ace-DEX of various dextran MWs and CAC was dissolved in hexafluoroisopropanol ( $2 \% \mathrm{vol} / \mathrm{wt}$ poly). The solution was dropped onto a silicon wafer (test grade, University Wafer). The wafer was placed on the spin coater (Chemat Technology KW-4A Spin Coater, Northridge, CA) and rotated at $500 \mathrm{rpm}$ for $5 \mathrm{~s}$ and at $2500 \mathrm{rpm}$ for another $60 \mathrm{~s}$. The wafer was air dried for $30 \mathrm{~min}$ at room temperature before water $(15 \mu \mathrm{L})$ was added using the sessile drop method. Images were obtained immediately using a ThorLabs camera (DCC1645C; Newton, NJ) and analyzed using Image to determine the contact angle.

\subsection{Preparation of blank or resiquimod-loaded Ace-DEX MPS}

Ace-DEX MPs were fabricated using a single-emulsion oil/water (o/w) evaporation method based on a modified protocol by Kauffman et al. (Kauffman et al., 2012b). In brief, Ace-DEX (100 mg) and resiquimod $(1 \mathrm{mg})$ were dissolved in ethyl acetate $(1 \mathrm{~mL})$ and added to poly(vinyl alcohol) $(2 \mathrm{~mL})(3 \% \mathrm{wt} / \mathrm{wt}$ PVA in phosphate buffered saline, PBS) in preparation of resiquimod-loaded Ace-DEX MPs. The mixture was vortexed for $5 \mathrm{~s}$ and probe sonicated for $30 \mathrm{~s}$ (amplitude 8,1 s pulse on, $1 \mathrm{~s}$ pulse off; Misonix Ultrasonic Liquid Processor, Newtown, CT). The emulsion was transferred to a small beaker containing 0.3\% PVA in PBS $(20 \mathrm{~mL})$ and stirred for $2 \mathrm{~h}$. The solution was centrifuged for $45 \mathrm{~min}$ at $14,400 \mathrm{rpm}$ at $4{ }^{\circ} \mathrm{C}$. The particle sediment was washed twice with basic water and lyophilized for 2 days. Blank MPs were fabricated following the same procedure, except no resiquimod was added. Both blank and resiquimod-load MPs were made using polymers of different CAC and MW.

\subsection{Encapsulation efficiency of resiquimod}

Resiquimod encapsulation efficiency was determined as previously described (Duong et al., 2013). Briefly, samples of each resiquimod-loaded Ace-DEX MP set were prepared in triplicates and dissolved in dimethyl sulfoxide (DMSO). The solution was loaded onto a solvent-resistant 96-well plate together with a calibration curve containing free resiquimod. The amount of resiquimod was quantified based on its autofluorescence (260$360 \mathrm{~nm}$ ) using a SpectraMax M2 microplate reader (Molecular
Devices, Sunnyvale, CA). Blank MPs with corresponding MW and CAC were used to measure the background signal.

\subsection{Size determination of Ace-DEX MPs}

The average diameter of Ace-DEX MPs was measured by Mean Diameter by Number using dynamic light scattering (DLS) following a previously described protocol (Duong et al., 2013). Ace-DEX MPs were resuspended in basic water $(33.3 \mu \mathrm{g} / \mathrm{mL})$ and sized using a Brookhaven NanoBrook 90Plus Zeta Particle Size Analyzer (Holtsville, NY).

\subsection{Scanning electron microscopy (SEM)}

Ace-DEX MPs were observed and characterized by; Hitachi s4300 Cold Field Emission. SEM samples were prepared by mounting MPs directly onto the SEM pin stub using carbon tape. The samples were sputter-coated with a $7 \mathrm{~nm}$ layer of palladium/ gold alloy before imaging (Kauffman et al., 2012a; Kauffman et al., 2012b).

\subsection{Endotoxin analysis}

All Ace-DEX MPs were prepared using endotoxin free beakers and stir bars, which were cleaned by soaking in $1 \mathrm{M}$ sodium hydroxide solution overnight. Solutions were prepared fresh on the day of particle fabrication. An endotoxin assay was conducted to confirm the absence of endotoxin of all Ace-DEX MPs. To perform the assay, MPs were resuspended in nanopure water $(1 \mathrm{mg} / \mathrm{mL})$ and incubated at $4{ }^{\circ} \mathrm{C}$ overnight. They were centrifuged at $14,800 \mathrm{rpm}$ for $10 \mathrm{~min}$, and supernatant was used for the assay using the Pierce LAL Chromogenic Endotoxin Quantitation Kit following the manufacturer's directions (Thermo Scientific, Waltham, MA).

\subsection{Degradation analysis of Ace-DEX MPs by optical density measurement}

Blank Ace-DEX MPs with different CAC and MWs were resuspended in either $\mathrm{PBS}(\mathrm{pH}=7.4)$ or $0.3 \mathrm{M}$ sodium acetate buffer ( $\mathrm{pH} 5.0)$ in triplicates $(1.5 \mathrm{mg} / \mathrm{mL})$. Samples were agitated on a shaker plate $(150 \mathrm{rpm})$ at $37^{\circ} \mathrm{C}$. At $0,0.5,2,4,8,24,48,72,168$, and $336 \mathrm{~h}$, the solution was vortexed, and its absorbance at $600 \mathrm{~nm}$ was read using a SpectraMax M2 microplate reader (Molecular Devices, Sunnyvale, CA). The degradation profile of MPs was determined by comparing their absorbance levels at different time points to that at $0 \mathrm{~h}$. The percent degradation of each sample is equal to the difference between its absorbance reading and that at $0 \mathrm{~h}$ divided by the absorbance reading at $0 \mathrm{~h}$. The degradation halflife was defined as the time when $50 \%$ of the MPs had degraded.

\subsection{Degradation analysis of Ace-DEX MPs by bicinchoninic acid based assay}

Blank $10 \mathrm{kDa}$ Ace-DEX MPs (20\%, 40\%, and 60\%) were resuspended in either $\mathrm{PBS}(\mathrm{pH}=7.4)$ or $0.3 \mathrm{M}$ sodium acetate buffer ( $\mathrm{pH} 5.0$ ) in triplicates $(1.5 \mathrm{mg} / \mathrm{mL}$ ). Samples were agitated on a shaker plate $(150 \mathrm{rpm})$ at $37^{\circ} \mathrm{C}$. At $0,0.5,2,4,8,24,48$ and $72 \mathrm{~h}$ the solution was vortexed, and an aliquot $(120 \mu \mathrm{L})$ was collected. The aliquots were centrifuged at $14,800 \mathrm{rpm}$ for $15 \mathrm{~min}$. Supernatant $(100 \mu \mathrm{L})$ was transferred to a 96 -well polystyrene plate and stored at $-20^{\circ} \mathrm{C}$. After the last time point, samples were analyzed using a microplate reductometric bicinchoninic acid based assay (BCA) following the manufacturer's guideline (Micro BCA Protein Assay Kit; Thermo Scientific, Waltham, MA). This assay measures the amount of degradation product dextran in the supernatant 
over time. The percent degradation of each sample was obtained by normalizing its reading at a certain time point to that of the respective MP set at $\mathrm{pH} 5$ at the last time point when it was fully degraded.

\subsection{Release kinetics of resiquimod-loaded Ace-DEX MPS}

Resiquimod-loaded Ace-DEX MPs with different CAC and MW were resuspended in either PBS $(\mathrm{pH}=7.4)$ or $0.3 \mathrm{M}$ sodium acetate buffer ( $\mathrm{pH} 5.0)$ in triplicates $(1.5 \mathrm{mg} / \mathrm{mL})$. Samples were agitated on a shaker plate $(150 \mathrm{rpm})$ at $37^{\circ} \mathrm{C}$. At $0.5,2,4,8,24,48,72,168$, and $336 \mathrm{~h}$, the solution was vortexed, and an aliquot $(170 \mu \mathrm{L})$ was collected. The aliquots were centrifuged at $14,800 \mathrm{rpm}$ for $15 \mathrm{~min}$. Supernatant $(150 \mu \mathrm{L})$ was transferred to a 96 -well polystyrene plate, combined with $30 \mu \mathrm{L}$ ethanol, and stored at $-20^{\circ} \mathrm{C}$. At the last time point (hour 336$)$, a sample $(150 \mu \mathrm{L})$ was removed from the homogenous suspension and treated with $30 \mu \mathrm{L}$ ethanol to degrade any remaining MPs. This sample contained the total amount of resiquimod (100\% released). The amount of resiquimod in the supernatant was quantified based on its autofluorescence (260-360 nm) using a SpectraMax M2 microplate reader (Molecular Devices, Sunnyvale, CA). The percentage of resiquimod released was calculated relative to the fluorescence of the respective MP set after complete degradation at the last time point.

\subsection{Cell viability analysis}

Viability of RAW 264.7 macrophages after incubation with different Ace-DEX MPs was studied using a 3-(4,5-dimethylthiazol-2-yl)-2,5-diphenyltetrazolium bromide (MTT) assay. Cells were seeded overnight in a 96-well plate at a density of 40,000 cells per well. Various Ace-DEX MPs $(10,71,500$, or $2000 \mathrm{kDa}$ with $20 \%, 40 \%$, or $60 \%$ CAC) were resuspended in culture media and added on the next day $(0.0625,0.125,0.25,0.5$, and $1 \mathrm{mg} / \mathrm{mL})$. After 24 or $48 \mathrm{~h}$ incubation, MTT assay was performed to examine cell viability. Briefly, culture media in each well was replaced with a solution of MTT reagent in fresh media $(0.6 \mathrm{mg} / \mathrm{mL}, 170 \mu \mathrm{L})$. The plate was incubated at $37^{\circ} \mathrm{C}$ for around $1 \mathrm{~h}$ until purple formazan crystals formed. The supernatant was replaced with isopropanol $(200 \mu \mathrm{L})$, and the solution was pipetted up and down to dissolve the crystals. The plate was read at 560 and $670 \mathrm{~nm}$ using a plate reader. Background absorbance at $670 \mathrm{~nm}$ was subtracted from all $560 \mathrm{~nm}$ readings. Cell viability was determined by comparing the readings of the treated groups to those of the negative (mediaonly) and positive ( $10 \% \mathrm{v} / \mathrm{v}$ Tween 80 ) controls. Signals of positive controls were subtracted from all readings to obtain backgroundadjusted values. Percent viability was calculated to be the result of the adjusted-reading for MP-treated cells divided by the adjustedreading for the negative control.

\subsection{Cytotoxicity analysis}

Cytotoxicity of various Ace-DEX MPs on RAW 264.7 macrophages was evaluated using a lactate dehydrogenase (LDH) assay (Pierce LDH Cytotoxicity Assay Kit; Thermo Scientific, Waltham, MA). Cells were seeded overnight in a 96-well plate at a density of 40,000 cells per well. Ace-DEX MPs $(10,71,500$, or $2000 \mathrm{kDa}$ with $20 \%, 40 \%$, or $60 \%$ CAC) were resuspended in culture media and added on the next day $(0.0625,0.125,0.25,0.5$, and $1 \mathrm{mg} / \mathrm{mL})$. After 24 or $48 \mathrm{~h}$ incubation, $100 \mu \mathrm{L}$ media was transferred to a 96 -well Vbottom plate, which was then centrifuged at $4200 \mathrm{rpm}$ for $10 \mathrm{~min}$ at room temperature. Supernatant $(50 \mu \mathrm{L})$ was transferred to a solvent-resistant 96-well microplate and analyzed in accordance with the manufacturer's protocol.

\subsection{Nitrite production analysis}

Bioactivity of various resiquimod-loaded Ace-DEX MPs on RAW 264.7 macrophages was evaluated using the nitrite production assay (Griess Reagent System; Promega, Madison, WI). Cells were seeded overnight in a 96-well plate at a density of 40,000 cells per well. Resiquimod-loaded Ace-DEX MPs (10, 71, 500, or 2000 kDa with $20 \%, 40 \%$, or $60 \%$ CAC) were resuspended in culture media and added on the next day. Particle concentrations (around $0.21 \mathrm{mg} /$ $\mathrm{mL}$ ) were adjusted based on their resiquimod encapsulation efficiency so that the final resiquimod concentration was the same across all groups $(0.15 \mu \mathrm{g} / \mathrm{mL})$. Media-only, free resiquimod $(0.15 \mu \mathrm{g} / \mathrm{mL})$, and lipopolysaccharide (LPS, $100 \mathrm{ng} / \mathrm{mL}$ ) were included as controls. Blank Ace-DEX (10 kDa, 60\%) MPs were included as a negative control. This MP set was selected because its correspondent resiquimod-loaded MP set required the highest particle concentration $(0.23 \mathrm{mg} / \mathrm{mL})$ in order to achieve the same resiquimod dose due to its low encapsulation efficiency. After 24 or $48 \mathrm{~h}$ incubation, $100 \mu \mathrm{L}$ media was transferred to a 96-well Vbottom plate, which was then centrifuged at $4200 \mathrm{rpm}$ for $10 \mathrm{~min}$ at room temperature. The supernatant $(50 \mu \mathrm{L})$ was transferred to a solvent-resistant 96 -well microplate and analyzed following the manufacturer's instructions.

\section{Results and discussion}

\subsection{Synthesis and characterization of Ace-DEX polymer and MPS}

We targeted $20 \%, 40 \%$, and $60 \%$ CAC for Ace-DEX polymers with different dextran starting material MWs $(10,71,500$, and $2000 \mathrm{kDa}$ ). The actual CAC values for Ace-DEX polymers synthesized for different periods of time are listed in Table 1. Because the actual CAC typically agreed with the target CAC, for the sake of clarity, the polymers will be referred to using the target CAC $(20 \%$, $40 \%$, or $60 \%$ ). For example, the shortest reaction $10 \mathrm{kDa}$ Ace-DEX polymer will be referred to as Ace-DEX (10 kDa, 20\%) instead of Ace-DEX (10 kDa, 18\%).

Water contact angle measurements of Ace-DEX polymers with varying CAC and MW are shown in Fig. 1. There was no strong correlation between the water contact angle and either polymer CAC or dextran MW. Ace-DEX polymers with varying CAC and dextran MW had comparable water contact angles, which suggested similar levels of hydrophobicity. The contact angles of Ace-DEX polymers are similar to those of PBAEs. Metter et al. showed that PBAEs synthesized from isobutylamine and 1,4butanediol diacrylate had a contact angle of $66.7^{\circ}$, while PBAEs synthesized from isobutylamine and poly(ethylene glycol)-200 diacrylate or diethylene glycol diacrylate showed a contact angle of $16.2^{\circ}$ or $35.5^{\circ}$, respectively (Metter et al., 2010). Both Ace-DEX and

Table 1

Relative cyclic acetal coverage (CAC) analysis of acetalated dextran polymer (AceDEX) synthesized from dextran with various molecular weights (MW) as determined by $1 \mathrm{H}$ NMR.

\begin{tabular}{llll}
\hline Dextran MW $(\mathrm{kDa})$ & Reaction Time $(\mathrm{min})$ & Target CAC $(\%)$ & Actual CAC $(\%)$ \\
\hline 10 & 5.2 & 20 & 18 \\
& 52.6 & 40 & 41 \\
71 & 1440 & 60 & 61 \\
& 3.2 & 20 & 19 \\
500 & 25 & 40 & 37 \\
& 1560 & 60 & 61 \\
& 4.4 & 20 & 20 \\
2,000 & 10 & 40 & 36 \\
& 166 & 60 & 59 \\
& 13.8 & 40 & 43 \\
& 55.2 & 60 & 60 \\
\hline
\end{tabular}




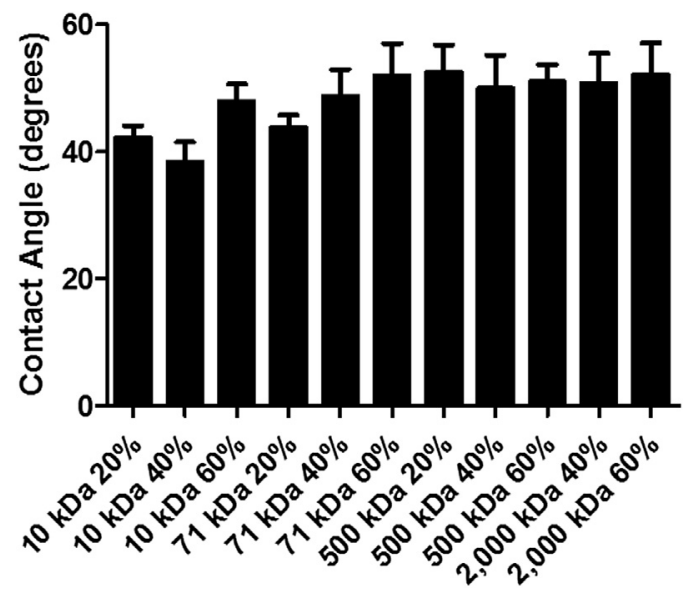

Fig. 1. Water contact angles of acetalated dextran polymers of various molecular weight of dextran starting material and relative cyclic acetal coverage (\%). Data are presented as mean \pm standard deviation $(n=5)$.

PBAEs are less hydrophobic than PLGA, whose water contact angle is $98^{\circ}$ (Zhang and Webster, 2013).

Ace-DEX polymers of varying CAC and MW were than formulated into MPs, which were characterized for size and morphology using DLS (Table 2) and SEM (Fig. 2). SEM images of blank Ace-DEX (40\% CAC) MPs of varying MW (10, 71, 500, and $2000 \mathrm{kDa}$ ) can be found in Fig. 2. SEM images of MPs formulated using other CAC $(20 \%$ or $60 \%$ ) can be found in Fig. S1. The SEM micrographs highlight that the MPs' spherical morphology was not affected by CAC or dextran MW.

To study the degradation profile of Ace-DEX MPs and its relationship to polymer CAC and dextran MW, we incubated blank MPs at $\mathrm{pH} 7.4$ and 5.0. Table 3 displays the degradation half-lives of eleven MP sets of varying CAC and MW. For MPs with the same dextran MW, degradation half-life related positively with CAC. Higher MW MPs underwent slower hydrolysis and demonstrated longer half-lives. For example, the half-life of Ace-DEX $(500 \mathrm{kDa}$, $20 \%$ ) MPs was $14.5 \mathrm{~h}$, shorter than $85.5 \mathrm{~h}$ of Ace-DEX (500 kDa, 40\%) MPs. The half-life of Ace-DEX (500 kDa, 60\%) MPs is reported as greater than $336 \mathrm{~h}$ (the last measurement) in Table 3 because less than $50 \%$ of the MPs were degraded at this final time point. The correlation between half-life and CAC was expected based on previous studies (Kauffman et al., 2012a), and can be explained by the greater hydrolytic stability of the thermodynamically-favored cyclic acetals compared to kinetically-favored acyclic acetals.

When CAC was held constant, degradation half-life of Ace-DEX MPs was inversely related to dextran MW (Table 3). Higher MW MPs underwent faster hydrolysis and had shorter half-lives. For

\section{Table 2}

Number-weighted mean diameter of blank acetalated dextran (Ace-DEX) microparticles (MPs) as determined by dynamic light scattering. MPs were composed of Ace-DEX polymers of different relative cyclic acetal coverage (CAC) and synthesized from dextrans of different molecular weights (MW). Data are presented as mean \pm standard deviation $(n=5)$.

\begin{tabular}{lll}
\hline Dextran MW (kDa) & Ace-DEX CAC $(\%)$ & Mean Diameter $(\mathrm{nm})$ \\
\hline 10 & 20 & $149 \pm 67$ \\
& 40 & $136 \pm 15$ \\
& 60 & $128 \pm 7$ \\
71 & 20 & $180 \pm 31$ \\
& 40 & $130 \pm 25$ \\
500 & 60 & $148 \pm 15$ \\
& 20 & $140 \pm 50$ \\
\multirow{2}{*}{2,000} & 40 & $242 \pm 68$ \\
& 60 & $230 \pm 51$ \\
& 40 & $159 \pm 49$ \\
\hline
\end{tabular}

example, the half-life of Ace-DEX (10 kDa, 20\%) MPs was $58.6 \mathrm{~h}$, much longer than $28.2 \mathrm{~h}$ of Ace-DEX ( $71 \mathrm{kDa}, 20 \%$ ) or $14.5 \mathrm{~h}$ of AceDEX (500 kDa, 20\%) MPs. This trend may be due to different packing densities of polymers with various MWs. Because dextran with smaller MWs has shorter chains, MPs made from Ace-DEX of lower dextran MWs were presumably more densely packed. This makes it harder for water molecules to diffuse into the polymer matrix, which results in a smaller area being exposed to water and slower MP hydrolysis. The same trend was observed for PLGA polymers. Wu et al. suggested that MPs made from PLGA polymers of higher MW degrade faster because their longer polymer chains are more likely to get hydrolyzed compared to their lower MW counterparts. The hydrolysis process was further accelerated due to autocatalysis of the newly-generated carboxylic end groups ( $\mathrm{Wu}$ and Wang, 2001).

Another hypothesis for the correlation between degradation rate and dextran MW might be irregular acetalation. Typical cyclic acetal groups form by replacement of two hydroxyl groups on a single glucose ring. Irregular acetalation could occur when hydroxyl groups of different glucose units (within or between dextran molecules) are modified. This may lead to cross-linking between separate dextran molecules and entanglement of the polymer, which leads to changes in its hydrolytic profile. If different glucose rings of the same dextran molecule are crosslinked, the Ace-DEX polymer may fold back on itself and become more loosely packed. When glucose rings of different dextran molecules are cross-linked, it may be harder for the irregularly linked chains to pack densely. We hypothesized that both processes might accelerate the hydrolysis process. Because polymers synthesized from higher MW dextran have longer chains, they are affected by the folding to a greater extent, which leads to faster degradation. Future experiments are needed in order to confirm this hypothesis. This correlation between AceDEX degradation rate and dextran MW was different than expected based on previous findings. Instead of a positive relationship between the two, Kauffman et al. previously showed that MPs composed of $10 \mathrm{kDa}$ Ac-DEX degraded faster than those composed of $71 \mathrm{kDa}$ Ac-DEX (Kauffman et al., 2012b). This could be explained by different properties of Ac-DEX and Ace-DEX. Although Ace-DEX shares many characteristics with its analog Ac-DEX, Ace-DEX has an additional carbon on its acyclic acetal groups, which introduces additional hydrophobicity and steric hindrance, possibly leading to different degradation profiles.

Besides neutral $\mathrm{pH}$, degradation of Ace-DEX MPs was also studied at $\mathrm{pH}$ 5.0, representative of the acidic condition within phagosomes of APCs (Ackerman and Simon, 2014; Walters and Papadimitriou, 1978), in order to illustrate the acid-sensitivity of the polymer. As seen in Table 3, MPs degraded much faster at $\mathrm{pH}$ 5.0 than at $\mathrm{pH}$ 7.4. Hydrolysis occurred so rapidly for some Ace-DEX (10, 71, $500 \mathrm{kDa} 20 \%, 500$ and $2000 \mathrm{kDa} 40 \%$ ) MPs that they were nearly, if not completely, fully degraded by the first measurement $(0.5 \mathrm{~h})$. As a result, their half-lives (around $0.25 \mathrm{~h}$ ) were much shorter compared to those at $\mathrm{pH}$ 7.4. Even the slowest-degrading Ace-DEX (10 kDa, 60\%) MP set had a half-life of $21.9 \mathrm{~h}$, much shorter than that of its corresponding group at $\mathrm{pH} 7.4(>336 \mathrm{~h})$. This faster degradation rate at $\mathrm{pH} 5.0$ agreed with previous studies and confirmed the acid sensitivity of acetal hydrolysis (Kauffman et al., 2012a). This acid-sensitive degradation is advantageous since it enables quick, localized release of encapsulated therapeutics in acidic environments for any of the previously mentioned applications.

To confirm the accuracy of the optical density measurement, degradation characteristics of Ace-DEX (10 kDa 20\%, 40\%, and 60\%) MPs were also examined using the BCA assay. Degradation profiles generated by the BCA assay qualitatively and quantitatively overlapped with those generated by optical density measurements 

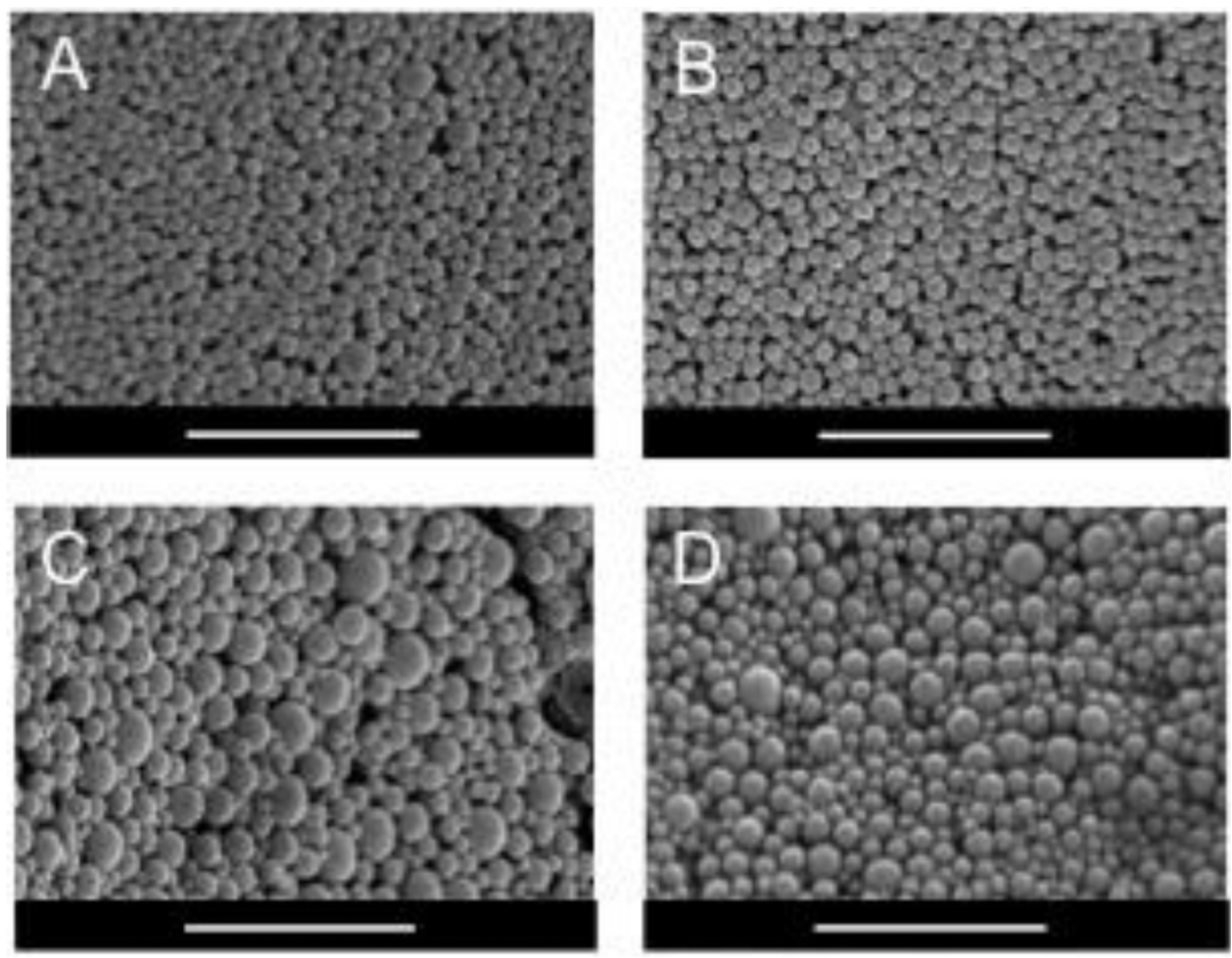

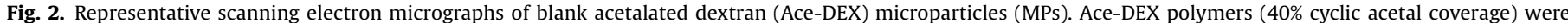
synthesized from various dextran molecular weights (MW): (A) $10 \mathrm{kDa}$, (B) $71 \mathrm{kDa},(\mathrm{C}) 500 \mathrm{kDa}$, and (D) $2000 \mathrm{kDa}$. Scale bars are $1 \mu \mathrm{m}$.

(Fig. S3), which indicated consistency of the two methods. Optical density assay was used for all degradation measurements in this study because it provided more reliable readings across different $\mathrm{pH}$ levels and could be used for polymers of different MWs. In the BCA assay, a copper ion reacts with only the reducing end group of the dextran molecule (Mann et al., 1992). Because polymers of higher dextran MW have larger units and fewer terminal groups, BCA was less reactive to Ace-DEX polymers with a MW larger than $10 \mathrm{kDa}$. For these reasons, the optical density measurement was used because it can be applied for varying dextran MWs across different $\mathrm{pH}$ levels. By studying the degradation profiles of AceDEX MPs, we concluded one advantage of this system to be the broad tunability as suggested by the wide range of degradation rates at both neutral and acidic pH. Degradation profiles can be precisely controlled and easily tuned for the desired therapeutic purpose by simply changing the dextran MW and/or CAC (i.e.,

Table 3

Degradation half-lives of blank microparticles (MPs) composed of acetalated dextran (Ace-DEX) with different dextran starting material molecular weights (MW) and relative cyclic acetal coverage (CAC) incubated at $\mathrm{pH} 7.4$ and 5.0. Data are presented as mean \pm standard deviation $(n=3)$. Half-lives of some MP sets are reported as greater than $336 \mathrm{~h}$, because less than 50\% MPs were degraded at this final time point.

\begin{tabular}{llll}
\hline Dextran MW (kDa) & Ace-DEX CAC (\%) & \multicolumn{2}{c}{ Blank MP Degradation Half-Life (hr) } \\
\cline { 3 - 4 } & & pH 7.4 & pH 5.0 \\
\hline \multirow{2}{*}{10} & 20 & $58.6 \pm 1.12$ & $0.26 \pm 0.0023$ \\
& 40 & $>336$ & $7.43 \pm 0.42$ \\
71 & 60 & $>336$ & $21.9 \pm 1.90$ \\
& 20 & $28.2 \pm 1.04$ & $0.25 \pm 0.00037$ \\
& 40 & $>336$ & $2.91 \pm 0.33$ \\
500 & 60 & $>336$ & $21.3 \pm 2.98$ \\
& 20 & $14.5 \pm 0.68$ & $0.25 \pm 0.00$ \\
& 40 & $85.5 \pm 0.80$ & $0.26 \pm 0.0013$ \\
2,000 & 60 & $>336$ & $21.0 \pm 0.53$ \\
& 40 & $48.6 \pm 2.23$ & $0.25 \pm 0.00080$ \\
\hline
\end{tabular}

reaction time) of the polymer. Flexibility of this system gives it a major advantage over other acid-sensitive polymers, such as PBAEs, POEs, and polyketals, of which degradation profiles are less variable.

Another potential advantage of Ace-DEX MPs is reduced cytotoxicity. Cytotoxicity of blank Ace-DEX MPs was analyzed by treating RAW macrophages with MPs of varying CAC and MW and evaluating it using both a MTT and LDH assay. A MTT and LDH were used because a MTT illustrates both viability and proliferation whereas a LDH assay provides cytotoxicity and cytolysis. In general, increasing MP concentrations led to lower MTT activity (Fig. 3A). Macrophages incubated for $48 \mathrm{~h}$ with low MP concentrations $(0.0625,0.125$, and $0.25 \mathrm{mg} / \mathrm{mL})$ showed high levels of cellular metabolic activities ( $>81 \%$ ) across all treatment groups (Fig. 3A). Macrophages treated with Ace-DEX (10 and $71 \mathrm{kDa}, 60 \%$ ) MPs at $1 \mathrm{mg} / \mathrm{mL}$ showed lower levels of metabolic activity. This dampened activity was observed previously by Reddy et al. where phagocytosis of a large number of apoptotic cells by macrophages led to inhibited proliferation (Reddy et al., 2002). Although MPs are not physiologically the same as apoptotic cells, we believe that phagocytosed MPs could result in similar outcomes (i.e., inhibited cell proliferation) as engulfed apoptotic cells. The LDH assay showed little to no cytotoxicity of Ace-DEX (10 and $71 \mathrm{kDa}$ ) MPs at all concentrations tested (Fig. 3B). For Ace-DEX MPs of higher MW (500 and $2000 \mathrm{kDa}$ ), the relationship between cell viability and MP concentration was less obvious based on the MTT assay. This might be because these higher MW MPs degraded faster under acidic conditions (Table 3 ), which resulted in macrophages being less saturated, allowing them to proliferate more. Although Ace-DEX (500 kDa, 60\%) MPs caused 31.3\% cell death according to the LDH assay (Fig. 3B), this should not be a cause for concern as this concentration $(1 \mathrm{mg} / \mathrm{mL})$ is much higher than what is practical for in vitro experiments (Peine et al., 2013; Peine et al., 2014a). Moreover, MPs formulated using PBAEs, which is also an acidsensitive polymer, exhibits much more severe cytotoxicity, as 

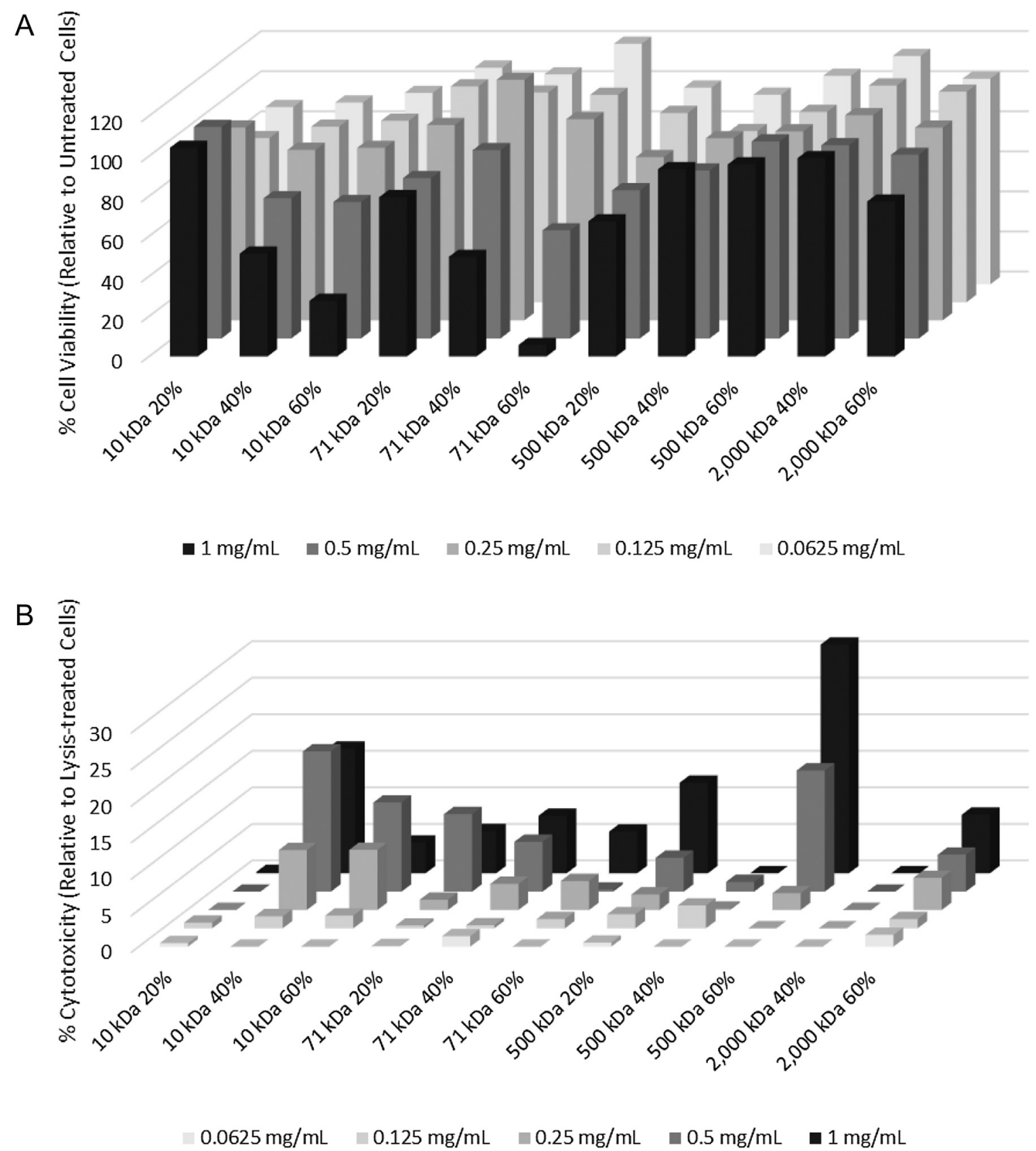

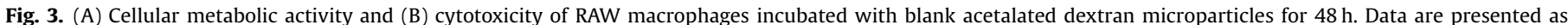
mean \pm standard deviation $(n=3)$. Groups are indicated by dextran molecular weight and relative cyclic acetal coverage of the acetalated dextran polymer.

$0.1 \mathrm{mg} / \mathrm{mL}$ PBAE MPs resulted in around 60\% death of P388D1 macrophages (Little et al., 2005). In a different study, $1 \mathrm{mg} / \mathrm{mL}$ PLGA MPs led to nearly $70 \%$ decrease in cellular metabolic activity in one of ours previous studies (Kauffman et al., 2012a). For a shorter, $24 \mathrm{~h}$ incubation period, RAW macrophages treated with Ace-DEX MPs of varying CAC and MW generated higher metabolic activity and showed similar trends as observed for the $48 \mathrm{~h}$ experiment (Fig. S4). Thus, we concluded that blank Ace-DEX MPs was well tolerated by macrophages under these experimental conditions.

\subsection{In vitro bioactivity of resiquimod-loaded Ace-DEX MPs}

After evaluating degradation profiles and cytotoxicity of blank Ace-DEX MPs of varying CAC and MW, we encapsulated resiquimod as a model drug to further characterize the system. Resiquimod is an immunomodulatory molecule used in vaccine formulations as a TLR 7/8 agonist. It has also been used for treatment of type 2 herpes simplex virus (HSV-2) and human papilloma (Mark et al., 2007). Unlike TLR 9 expression that is only limited to plasmacytoid dendritic cells in humans, TLR $7 / 8$ expression is similarly expressed in both mouse and humans, as well as other species (Applequist et al., 2002). Furthermore, because TLR 7/8 reside in the phagosome (Bishop et al., 2001; Borteh et al., 2013; Dockrell and Kinghorn, 2001; Duong et al., 2013), resiquimod is an ideal drug to study the effects of Ace-DEX MPs' degradation kinetics on drug bioactivity after Ace-DEX MPs are phagocytosed by macrophages (Hoang et al., 2014).

Resiquimod-loaded Ace-DEX MPs were characterized first for size and morphology using DLS (Table 4) and SEM (Fig. 4 and 
Table 4

Number-weighted mean diameter of resiquimod-loaded acetalated dextran (AceDEX) microparticles (MPs) as determined by dynamic light scattering. MPs were composed of Ace-DEX polymers of different relative cyclic acetal coverage (CAC) and synthesized from dextrans of different molecular weights (MW). Data are presented as mean \pm standard deviation $(n=5)$.

\begin{tabular}{lll}
\hline Dextran MW $(\mathrm{kDa})$ & Ace-DEX CAC $(\%)$ & Mean Diameter $(\mathrm{nm})$ \\
\hline 10 & 20 & $161 \pm 92$ \\
& 40 & $129 \pm 38$ \\
71 & 60 & $174 \pm 126$ \\
& 20 & $119 \pm 12$ \\
500 & 40 & $99.6 \pm 19$ \\
& 60 & $135 \pm 51$ \\
\multirow{2}{*}{2,000} & 20 & $\mathrm{~N} / \mathrm{A}$ \\
& 40 & $157 \pm 45$ \\
& 60 & $\mathrm{~N} / \mathrm{A}$ \\
& 40 & $156 \pm 87$ \\
& 60 & $\mathrm{~N} / \mathrm{A}$ \\
\hline
\end{tabular}

Fig. S2). Their average diameters were comparable to those of blank MPs (Table 2). The SEM micrographs of resiquimod-loaded MPs highlight that the MPs' spherical morphology was not affected by CAC, dextran MW, or resiquimod encapsulation.

Endotoxin levels and resiquimod encapsulation efficiency were then examined for these resiquimod-loaded Ace-DEX MPs. All MP sets had endotoxin levels below $0.25 \mathrm{EU} / \mathrm{mL}$ (Tables S1), which is the FDA's guideline for sterile water (U.S. Food and Drug Administration, 2015). Resiquimod encapsulation efficiency and final weight loading of Ace-DEX MPs are listed in Table 5. MPs with similar drug loading (0.65-1.0 $\mu$ g resiquimod per mg MP) were formulated for release, cyto-compatibility, and bioactivity studies. Having similar encapsulation efficiency across MP sets avoided the potential effect of drug loading on the release kinetics of resiquimod. Because the same resiquimod dose could be achieved using similar MP concentrations due to close drug loadings, a consistent particle to cell ratio across groups was ensured. This was important because higher particle to cell ratios has been associated with enhanced drug bioactivity (Duong et al., 2013; Jhunjhunwala et al., 2009).

Release kinetics of encapsulated resiquimod was characterized for Ace-DEX MPs of different CAC and MW. At pH 7.4, for MPs of the same dextran MW (10 and $71 \mathrm{kDa}$ ), resiquimod was released faster from MPs of lower CAC (20\% compared to 40\%) (Fig. 5A and B). This inverse relationship between cargo release and polymer CAC can be explained by the shorter degradation half-lives of MPs of lower CAC (Table 3). When polymer CAC was held constant (40\%), a higher level of resiquimod was released into the supernatant from higher MW MPs (Fig. 5C). This correlation between cargo release and polymer MW resulted from the faster hydrolysis of higher MW MPs (Table 3). Similar trends were observed under the acidic environment ( $\mathrm{pH}$ 5.0), where resiquimod was released faster from MPs of lower CAC or higher MW due to their faster degradation (Fig. 5). All MP sets released the drug faster at pH 5.0 compared to their counterpart under the $\mathrm{pH}$ neutral condition, suggesting acidsensitivity of Ace-DEX polymer. The difference between resiquimod release profiles among various MP sets was smaller under acidic conditions compared to that at a $\mathrm{pH}$ neutral environment. This may be due to the acid-sensitive nature of Ace-DEX polymer. Because all Ace-DEX MP sets degraded rapidly at pH 5.0 (Table 3), the effect of varying CAC or MW on resiquimod release was minimized. Therefore, release kinetics of encapsulated cargo can be tuned based on CAC and MW of Ace-DEX MPs, which makes Ace-DEX an ideal carrier for therapeutic delivery as it enables both rapid and sustained drug release via the same polymeric platform. When resiquimod was encapsulated into electrosprayed Ac-DEX (71 kDa) particles by Duong et al., a similar sustained release
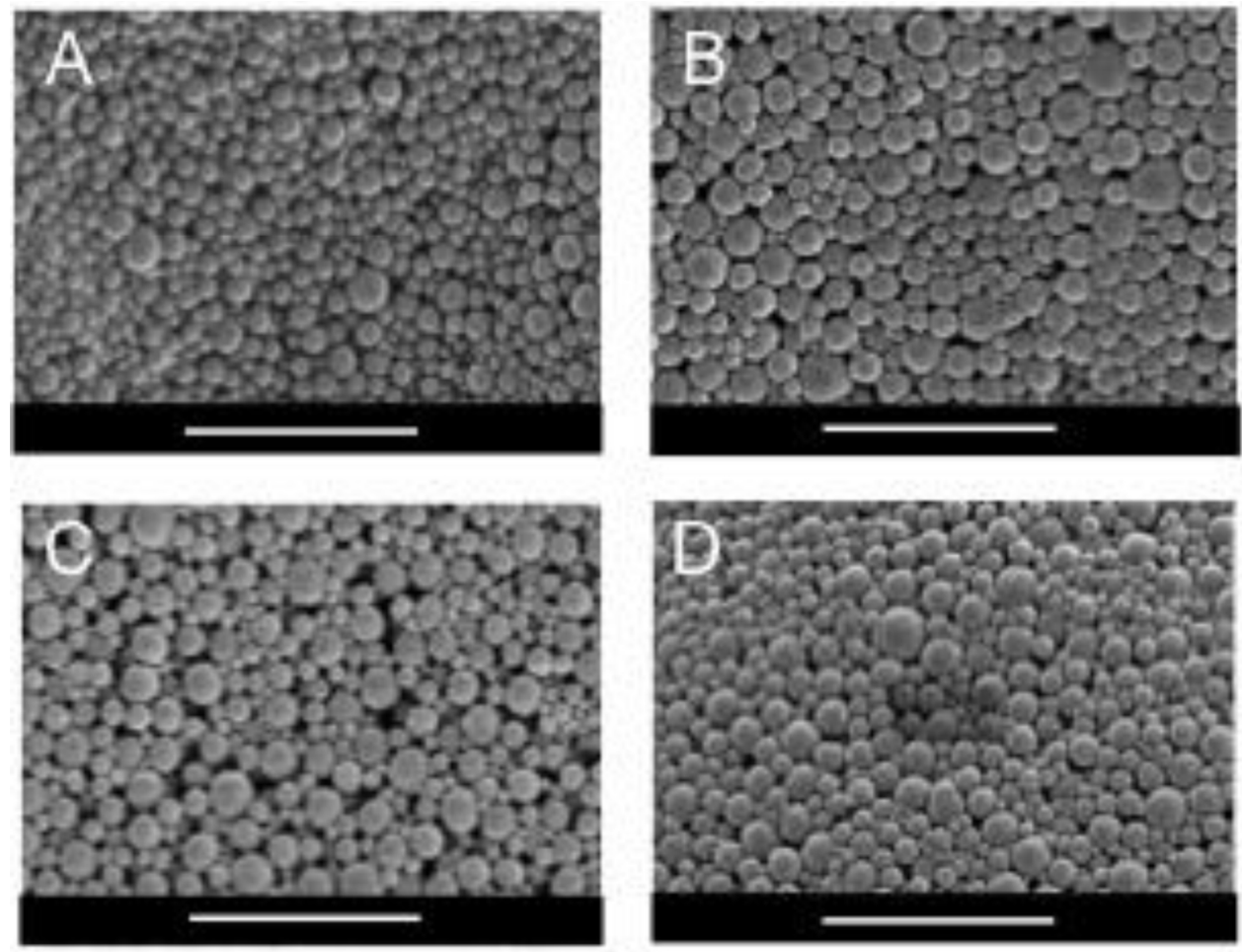

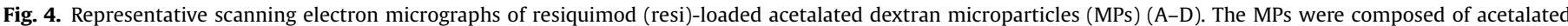

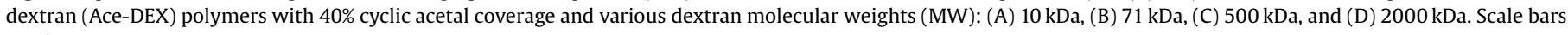
are $1 \mu \mathrm{m}$. 
Table 5

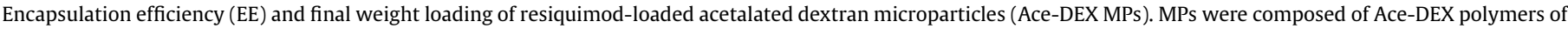
different relative cyclic acetal coverage $(C A C)$ and dextran molecular weights $(M W)$. Data are presented as mean \pm standard deviation $(n=3)$.

\begin{tabular}{|c|c|c|c|}
\hline Dextran MW (kDa) & Ace-DEX CAC (\%) & Resiquimod EE (\%) & Resiquimod Loading ( $\mu$ g drug/mg MP) \\
\hline \multirow[t]{3}{*}{10} & 20 & $6.70 \pm 0.77$ & $0.67 \pm 0.08$ \\
\hline & 40 & $6.78 \pm 0.36$ & $0.68 \pm 0.04$ \\
\hline & 60 & $6.45 \pm 1.91$ & $0.65 \pm 0.19$ \\
\hline \multirow[t]{3}{*}{71} & 20 & $6.54 \pm 1.03$ & $0.65 \pm 0.10$ \\
\hline & 40 & $7.38 \pm 1.24$ & $0.74 \pm 0.12$ \\
\hline & 60 & $7.64 \pm 1.06$ & $0.76 \pm 0.11$ \\
\hline 500 & 40 & $9.78 \pm 1.85$ & $0.98 \pm 0.19$ \\
\hline 2,000 & 40 & $6.69 \pm 4.24$ & $0.67 \pm 0.42$ \\
\hline
\end{tabular}

profile was observed with the compound released faster at $\mathrm{pH} 7.4$ (Duong et al., 2013). This discrepancy may be due to differences in fabrication methods and varying drug weight loading.

After evaluating the tunable and acid-sensitive release kinetics of resiquimod-loaded MPs, their cytotoxicity was analyzed on RAW macrophages using a MTT and LDH assay. For the $24 \mathrm{~h}$ incubation, all treatment groups showed comparable viability and proliferation levels to cells treated with blank MPs (Fig. 6A). Except for the LPS-treated positive control, all groups showed larger than 100\% metabolic activity, which was due to increased proliferation. The higher proliferation level was previously observed by Peine et al., where RAW macrophages were treated with resiquimod-loaded liposomes (Peine et al., 2014b). For the $48 \mathrm{~h}$ incubation, treatment groups showed lower metabolic activity than that observed for blank MPs, which might result from limited proliferation. Little to no cytotoxicity was observed for either treatment period as measured by the LDH assay (Fig. 6B). Thus, resiquimod-loaded AceDEX MPs exhibited similar levels of cyto-compatibility to blank MPs, which were both better than previously reported with PBAE MPs, causing a 60\% drop in the viability of P388D1 macrophages at $0.1 \mathrm{mg} / \mathrm{mL}$ (Little et al., 2005).

We then evaluated the in vitro bioactivity of resiquimod-loaded Ace-DEX MPs by monitoring the release of pro-inflammatory nitrite by RAW macrophages. Resiquimod-loaded Ace-DEX MPs of varying CAC and MW resulted in significantly higher levels of nitrite production with respect to blank MP-treated controls (Fig. 7). For cells incubated with $10 \mathrm{kDa}$ MPs of different CAC, lower nitrite production was observed with higher CAC. This inverse relationship between nitrite production and polymer CAC can be explained by different release kinetics of MPs with varying CAC (Fig. 5). Because MPs of higher CAC hydrolyzed slower under acidic conditions (Table 3 ), they likely released a smaller intraphagosomal amount of resiquimod after phagocytosis, resulting in less robust nitrite production. This trend was less obvious for $71 \mathrm{kDa}$ MPs because $71 \mathrm{kDa}$ Ace-DEX degraded faster than $10 \mathrm{kDa}$. By the end of the incubation period, most of the $71 \mathrm{kDa}$ MPs that had been phagocytosed by macrophages were nearly completely degraded, minimizing the effect of varying CAC.

When polymer CAC was held constant (40\%), nitrite production was in general more potent for higher MW MPs. MPs fashioned from 500 or $2000 \mathrm{kDa}$ dextran induced greater nitrite production than those formulated using polymer of lower MW. This trend may be due to different release profiles of resiquimod from MPs composed of varying dextran MW. A larger amount of resiquimod may be released after phagocytosis for higher MW MPs due to their faster hydrolysis (Table 3 and Fig. 5), resulting in more robust nitrite production. Therefore, macrophage activation and nitrite production can be precisely controlled by CAC and MW of Ace-DEX MPs, which makes Ace-DEX an ideal carrier for therapeutic delivery.
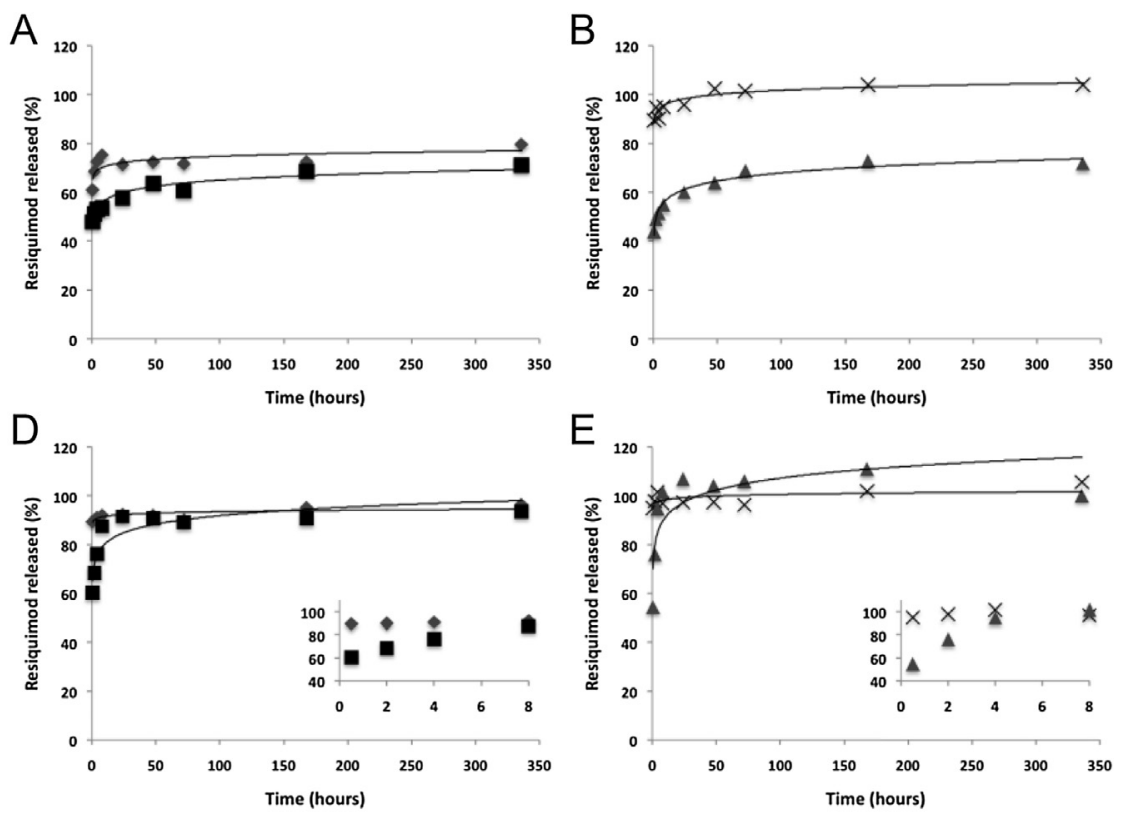

E
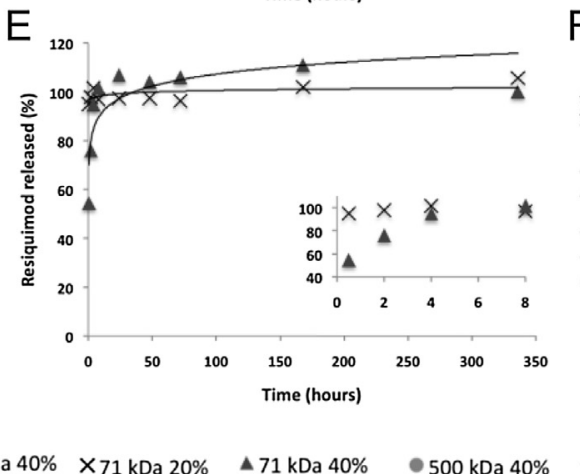

$\downarrow 10 \mathrm{kDa} 20 \%$

$10 \mathrm{kDa} 40 \% \times 71 \mathrm{kDa} 20 \% \quad \triangle 71 \mathrm{kDa} 40 \%$

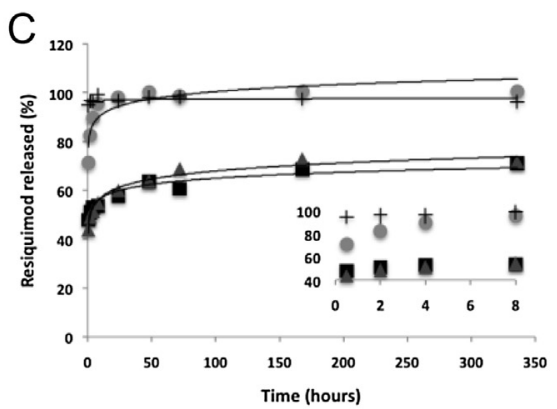

F

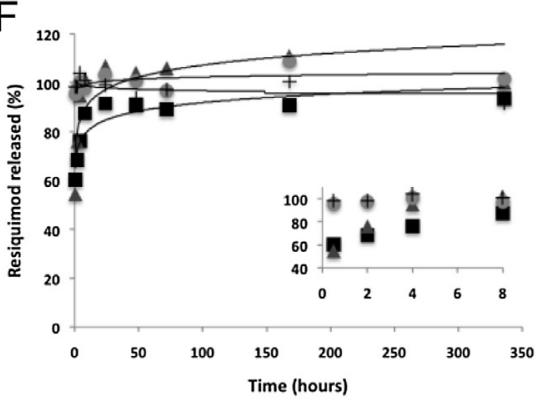

$500 \mathrm{kDa} 40 \% \quad+2000 \mathrm{kDa} 40 \%$

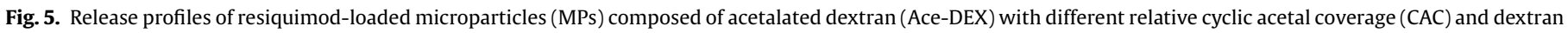
starting material molecular weights (MW) incubated at pH 7.4 (A-C) and 5.0 (D-F). The solid lines are sigmoidal functions added as a visual guide. 
A

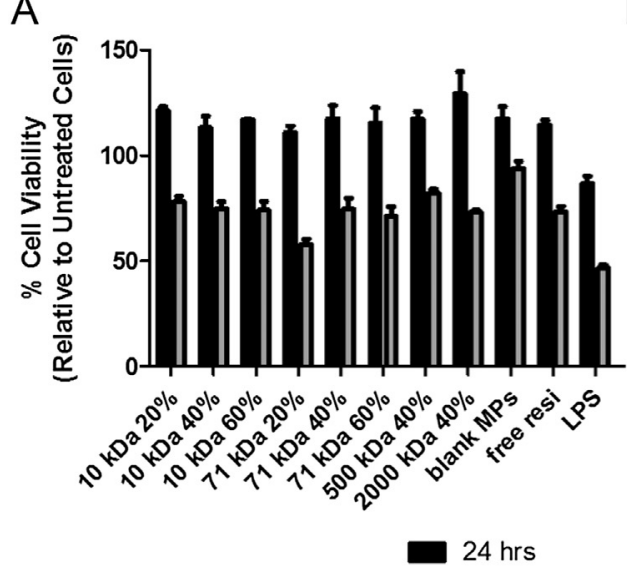

B

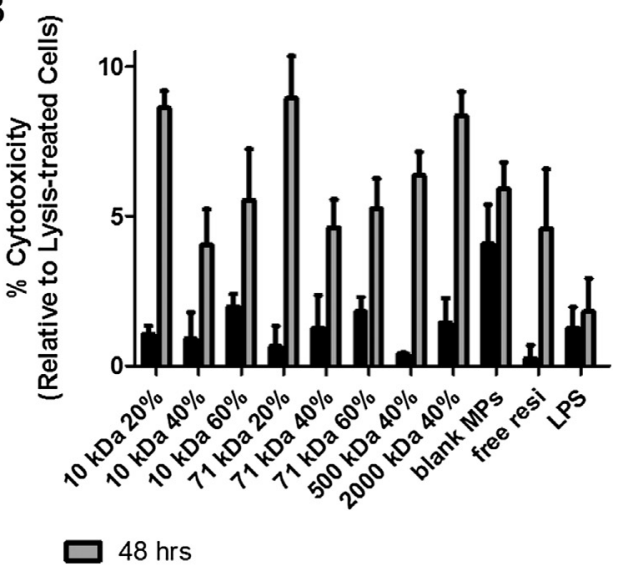

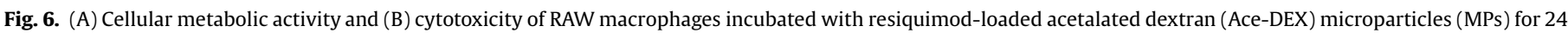

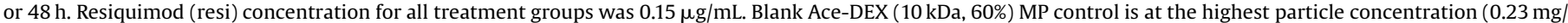
$\mathrm{mL}$ ). Lipopolysaccharide (LPS, $100 \mathrm{ng} / \mathrm{mL}$ ) was used as the positive control. Data are presented as mean \pm standard deviation $(\mathrm{n}=3$ ).

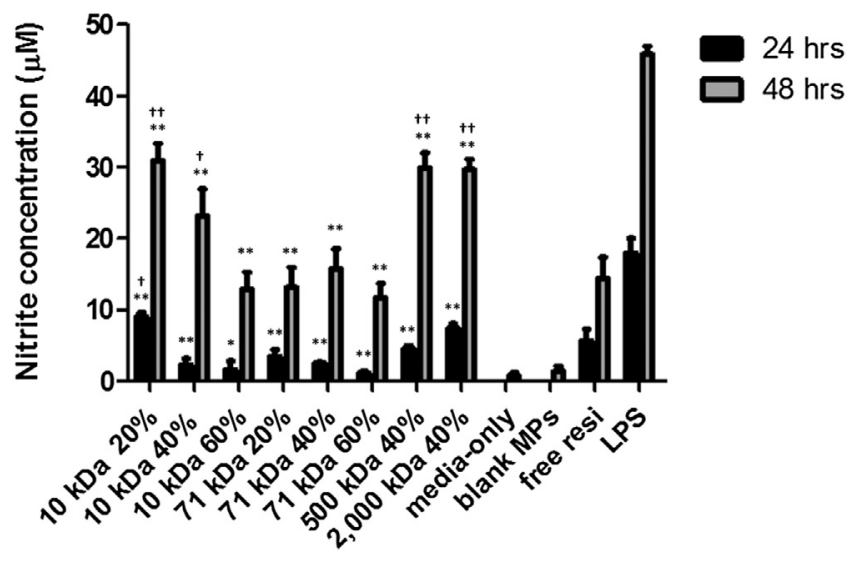

Fig. 7. Nitrite production by RAW macrophages incubated with free or encapsulated resiquimod (resi) after 24 or $48 \mathrm{~h}$. Resiquimod was encapsulated by acetalated dextran (Ace-DEX) microparticles (MPs) with varying dextran molecular weight and relative cyclic acetal coverage. Resiquimod concentration for all treatment groups was $0.15 \mu \mathrm{g} / \mathrm{mL}$. Blank Ace-DEX (10 kDa, 60\%) MP control is at the highest particle concentration $(0.23 \mathrm{mg} / \mathrm{mL}$ ). Lipopolysaccharide (LPS, $100 \mathrm{ng} / \mathrm{mL}$ ) was used as the positive control. Data are presented as mean \pm standard deviation $(n=3)$. Statistical significance with respect to blank MPs is presented as ${ }^{*} \mathrm{p}<0.05$ and ${ }^{* *} \mathrm{p}<0.005$. Statistical significance with respect to free resiquimod is presented as $\dagger \mathrm{p}<0.05$ and $\dagger \dagger \mathrm{p}<0.005$.

\section{Conclusion}

We have shown that Ace-DEX MPs with lower CAC or higher dextran MW hydrolyzed faster under both physiologically neutral pH 7.4 and phagosomal pH 5.0, with degradation half-lives being much shorter under acidic conditions, compared to those with higher CAC or lower MW. The facile tunability of Ace-DEX chemistry allowed for both burst and sustained long-term release of encapsulated therapeutics using the same polymeric platform, which makes Ace-DEX an ideal material for particle formulation. Overall, acid-sensitivity, tunable degradation, pH neutral metabolites, and minimal toxicity on macrophages in culture make AceDEX advantageous over existing acid-sensitive polymeric systems and a promising candidate for particle formulation and therapeutic delivery. Future work exploring the degradation profiles of AceDEX polymers of varying CAC and MW at intermediate $\mathrm{pH}$ levels will further characterize the tunability of this platform. Scalable production and in vivo efficacy of Ace-DEX MPs will also be studied to further demonstrate the novelty and advantages of this polymer system.

\section{Acknowledgements}

This work was supported by Internal Funds of the University of North Carolina at Chapel Hill. The authors also thank Chapel Hill Analytical and Nanofabrication Laboratory for their aid in acquiring SEM images.

\section{Appendix A. Supplementary data}

Supplementary data associated with this article can be found, in the online version, at http://dx.doi.org/10.1016/j. ijpharm.2016.08.031.

\section{References}

Ackerman, D., Simon, M.C., 2014. Hypoxia, lipids, and cancer: surviving the harsh tumor microenvironment. Trends Cell Biol. 24, 472-478.

Applequist, S.E., Wallin, R.P., Ljunggren, H.G., 2002. Variable expression of toll-like receptor in murine innate and adaptive immune cell lines. Int. Immunol. 14, 1065-1074.

Bachelder, E.M., Beaudette, T.T., Broaders, K.E., Dashe, J., Frechet, J.M., 2008. Acetalderivatized dextran: an acid-responsive biodegradable material for therapeutic applications. J. Am. Chem. Soc. 130, 10494-10495.

Bishop, G.A., Ramirez, L.M., Baccam, M., Busch, L.K., Pederson, L.K., Tomai, M.A., 2001. The immune response modifier resiquimod mimics CD40-induced B cell activation. Cell. Immunol. 208, 9-17.

Borteh, H.M., Gallovic, M.D., Sharma, S., Peine, K.J., Miao, S., Brackman, D.J., Gregg, K., Xu, Y., Guo, X., Guan, J., Bachelder, E.M., Ainslie, K.M., 2013. Electrospun acetalated dextran scaffolds for temporal release of therapeutics. Langmuir 29, 7957-7965.

Broaders, K.E., Cohen, J.A., Beaudette, T.T., Bachelder, E.M., Frechet, J.M., 2009. Acetalated dextran is a chemically and biologically tunable material for particulate immunotherapy. Proc. Natl. Acad. Sci. U. S. A. 106, 5497-5502.

Dockrell, D.H., Kinghorn, G.R., 2001. Imiquimod and resiquimod as novel immunomodulators. J. Antimicrob. Chemother. 48, 751-755.

Duong, A.D., Sharma, S., Peine, K.J., Gupta, G., Satoskar, A.R., Bachelder, E.M., Wyslouzil, B.E., Ainslie, K.M., 2013. Electrospray encapsulation of toll-like receptor agonist resiquimod in polymer microparticles for the treatment of visceral leishmaniasis. Mol. Pharm. 10, 1045-1055.

Engin, K., Leeper, D.B., Cater, J.R., Thistlethwaite, A.J., Tupchong, L., McFarlane, J.D. 1995. Extracellular pH distribution in human tumours. Int. J. Hyperthermia 11, 211-216.

Fu, K., Pack, D.W., Klibanov, A.M., Langer, R., 2000. Visual evidence of acidic environment within degrading poly(lactic-co-glycolic acid) (PLGA) microspheres. Pharm. Res. 17, 100-106.

Hoang, K.V., Borteh, H.M., Rajaram, M.V., Peine, K.J., Curry, H., Collier, M.A., Homsy, M.L., Bachelder, E.M., Gunn, J.S., Schlesinger, L.S., Ainslie, K.M., 2014. Acetalated dextran encapsulated AR-12 as a host-directed therapy to control Salmonella infection. Int. J. Pharm. 477, 334-343. 
Hu, Y., Litwin, T., Nagaraja, A.R., Kwong, B., Katz, J., Watson, N., Irvine, D.J., 2007. Cytosolic delivery of membrane-impermeable molecules in dendritic cells using pH-responsive core-shell nanoparticles. Nano Lett. 7, 3056-3064.

Jeong, B., Gutowska, A., 2002. Lessons from nature: stimuli-responsive polymers and their biomedical applications. Trends Biotechnol. 20, 305-311.

Jhunjhunwala, S., Raimondi, G., Thomson, A.W., Little, S.R., 2009. Delivery of rapamycin to dendritic cells using degradable microparticles. J. Control Release 133, 191-197.

Ji, R., Cheng, J., Song, C., Du, F.S., Liang, D.H., Li, Z.C., 2014a. Acid-sensitive polypseudorotaxanes based on ortho ester-modified cyclodextrin and pluronic F-127. ACS 4, 5.

Ji, R., Cheng, J., Yang, T., Song, C.C., Li, L., Du, F.S., Li, Z.C., 2014b. Shell-sheddable, pHsensitive supramolecular nanoparticles based on ortho ester-modified cyclodextrin and adamantyl PEG. Biomacromolecules 15, 3531-3539.

Kanthamneni, N., Sharma, S., Meenach, S.A., Billet, B., Zhao, J.C., Bachelder, E.M., Ainslie, K.M., 2012. Enhanced stability of horseradish peroxidase encapsulated in acetalated dextran microparticles stored outside cold chain conditions. Int. J. Pharm. 431, 101-110.

Kauffman, K.J., Do, C., Sharma, S., Gallovic, M.D., Bachelder, E.M., Ainslie, K.M., 2012a. Synthesis and characterization of acetalated dextran polymer and microparticles with ethanol as a degradation product. ACS Appl. Mater Interfaces 4, 4149-4155.

Kauffman, K.J., Kanthamneni, N., Meenach, S.A., Pierson, B.C., Bachelder, E.M., Ainslie, K.M., 2012b. Optimization of rapamycin-loaded acetalated dextran microparticles for immunosuppression. Int. J. Pharm. 422, 356-363.

Lee, E.S., Gao, Z., Bae, Y.H., 2008. Recent progress in tumor pH targeting nanotechnology. J. Control. Release 132, 164-170.

Lee, S., Yang, S.C., Kao, C.Y., Pierce, R.H., Murthy, N., 2009. Solid polymeric microparticles enhance the delivery of siRNA to macrophages in vivo. Nucleic Acids Res. 37, e145.

Little, S.R., Lynn, D.M., Ge, Q., Anderson, D.G., Puram, S.V., Chen, J., Eisen, H.N., Langer, R., 2004. Poly-beta amino ester-containing microparticles enhance the activity of nonviral genetic vaccines. Proc. Natl. Acad. Sci. U. S. A. 101, 9534-9539.

Little, S.R., Lynn, D.M., Puram, S.V., Langer, R., 2005. Formulation and characterization of poly (beta amino ester) microparticles for genetic vaccine delivery. J. Control. Release 107, 449-462.

Makhlof, A., Tozuka, Y., Takeuchi, H., 2009. pH-Sensitive nanospheres for colonspecific drug delivery in experimentally induced colitis rat model. Eur. J. Pharm. Biopharm. 72, 1-8.

Mandracchia, D., Pitarresi, G., Palumbo, F.S., Carlisi, B., Giammona, G., 2004. pHsensitive hydrogel based on a novel photocross-linkable copolymer. Biomacromolecules 5, 1973-1982.

Mann, J.S., Huang, J.C., Keana, J.F., 1992. Molecular amplifiers: synthesis and functionalization of a poly(aminopropyl)dextran bearing a uniquely reactive terminus for univalent attachment to biomolecules. Bioconjugate Chem. 3 . 154-159.

Manolova, V., Flace, A., Bauer, M., Schwarz, K., Saudan, P., Bachmann, M.F., 2008. Nanoparticles target distinct dendritic cell populations according to their size. Eur. J. Immunol. 38, 1404-1413.

Mark, K.E., Corey, L., Meng, T.C., Magaret, A.S., Huang, M.L., Selke, S., Slade, H.B., Tyring, S.K., Warren, T., Sacks, S.L., Leone, P., Bergland, V.A., Wald, A., 2007. Topical resiquimod $0.01 \%$ gel decreases herpes simplex virus type 2 genital shedding: a randomized, controlled trial. J. Infect. Dis. 195, 1324-1331.
Mellman, I., Fuchs, R., Helenius, A., 1986. Acidification of the endocytic and exocytic pathways. Annu. Rev. Biochem. 55, 663-700.

Metter, R.B., Ifkovits, J.L., Hou, K., Vincent, L., Hsu, B., Wang, L., Mauck, R.L., Burdick, J. A., 2010. Biodegradable fibrous scaffolds with diverse properties by electrospinning candidates from a combinatorial macromer library. Acta Biomater. 6, 1219-1226.

Murthy, N., Thng, Y.X., Schuck, S., Xu, M.C., Frechet, J.M., 2002. A novel strategy for encapsulation and release of proteins: hydrogels and microgels with acid-labile acetal cross-linkers. J. Am. Chem. Soc. 124, 12398-12399.

Na, D.H., Youn, Y.S., Lee, S.D., Son, M.W., Kim, W.B., DeLuca, P.P., Lee, K.C., 2003. Monitoring of peptide acylation inside degrading PLGA microspheres by capillary electrophoresis and MALDI-TOF mass spectrometry. J. Control. Release 92, 291-299.

Paramonov, S.E., Bachelder, E.M., Beaudette, T.T., Standley, S.M., Lee, C.C., Dashe, J., Frechet, J.M., 2008. Fully acid-degradable biocompatible polyaceta microparticles for drug delivery. Bioconjugate Chem. 19, 911-919.

Peine, K.J., Bachelder, E.M., Vangundy, Z., Papenfuss, T., Brackman, D.J., Gallovic, M. D., Schully, K., Pesce, J., Keane-Myers, A., Ainslie, K.M., 2013. Efficient delivery of the toll-like receptor agonists polyinosinic:polycytidylic acid and $\mathrm{CpG}$ to macrophages by acetalated dextran microparticles. Mol. Pharm. 10, 2849-2857.

Peine, K.J., Guerau-de-Arellano, M., Lee, P., Kanthamneni, N., Severin, M., Probst, G. D., Peng, H., Yang, Y., Vangundy, Z., Papenfuss, T.L., Lovett-Racke, A.E., Bachelder E.M., Ainslie, K.M., 2014a. Treatment of experimental autoimmune encephalomyelitis by codelivery of disease associated peptide and dexamethasone in acetalated dextran microparticles. Mol. Pharm. 11, 828-835.

Peine, K.J., Gupta, G., Brackman, D.J., Papenfuss, T.L., Ainslie, K.M., Satoskar, A.R., Bachelder, E.M., 2014b. Liposomal resiquimod for the treatment of Leishmania donovani infection. J. Antimicrob. Chemother. 69, 168-175.

Reddy, S.M., Hsiao, K.H., Abernethy, V.E., Fan, H., Longacre, A., Lieberthal, W., Rauch, J., Koh, J.S., Levine, J.S., 2002. Phagocytosis of apoptotic cells by macrophages induces novel signaling events leading to cytokine-independent survival and inhibition of proliferation: activation of Akt and inhibition of extracellular signal-regulated kinases 1 and 2. J. Immunol. 169, 702-713.

Sawant, R.M., Hurley, J.P., Salmaso, S., Kale, A., Tolcheva, E., Levchenko, T.S., Torchilin, V.P., 2006. SMART drug delivery systems: double-targeted $\mathrm{pH}$-responsive pharmaceutical nanocarriers. Bioconjugate Chem. 17, 943-949.

Sung, H.J., Meredith, C., Johnson, C., Galis, Z.S., 2004. The effect of scaffold degradation rate on three-dimensional cell growth and angiogenesis. Biomaterials 25, 5735-5742.

Ulbrich, W., Lamprecht, A., 2010. Targeted drug-delivery approaches by nanoparticulate carriers in the therapy of inflammatory diseases. J. R. Soc. Interface 7 (Suppl. 1), S55-66.

Walters, M.N., Papadimitriou, J.M., 1978. Phagocytosis: a review. CRC Crit. Rev. Toxicol. 5, 377-421.

Wu, X.S., Wang, N., 2001. Synthesis, characterization, biodegradation, and drug delivery application of biodegradable lactic/glycolic acid polymers Part II: biodegradation. J. Biomater. Sci. Polym. Ed. 12, 21-34.

Yang, S.C., Bhide, M., Crispe, I.N., Pierce, R.H., Murthy, N., 2008. Polyketal copolymers: a new acid-sensitive delivery vehicle for treating acute inflammatory diseases. Bioconjugate Chem. 19, 1164-1169.

Zhang, L., Webster, T.J., 2013. Effects of chemically modified nanostructured PLGA on functioning of lung and breast cancer cells. Int. J. Nanomed. 8, 1907-1919. 